Revue d'histoire de l'Amérique française

REVUE D.HISTOIRE DE L'AMÉRIQUE FRANÇAISE

\title{
Louise Réaume-Fournerie-Robertson (1742-1773) et son petit-fils le Colonel Daniel de Hertel (1797-1866)
}

\section{Jean-Jacques Lefebvre}

Volume 12, numéro 3, décembre 1958

URI : https://id.erudit.org/iderudit/301917ar

DOI : https://doi.org/10.7202/301917ar

Aller au sommaire du numéro

Éditeur(s)

Institut d'histoire de l'Amérique française

ISSN

0035-2357 (imprimé)

1492-1383 (numérique)

Découvrir la revue

Citer cet article

Lefebvre, J.-J. (1958). Louise Réaume-Fournerie-Robertson (1742-1773) et son petit-fils le Colonel Daniel de Hertel (1797-1866). Revue d'histoire de l'Amérique française, 12(3), 323-334. https://doi.org/10.7202/301917ar d'utilisation que vous pouvez consulter en ligne. 


\section{LOUISE REAAUME-FOURNERIE-ROBERTSON (1742-1773)}

\section{ET \\ SON PETIT-FILS LE COLONEL DANIEL DE HERTEL (1797-1866)}

A la faveur de la folie de mariage qui sévit à Québec et à Montréal aux derniers jours de la Nouvelle-France, la montréalaise, Louise Réaume, ${ }^{1}$ née en 1742 , s'alliait, en 1760 , à un ingénieur du roi, Fournerie de Vezon. Entrée dans l'histoire avec l'analyse qu'Aegidius Fauteux fit, en $1916,{ }^{2}$ des bibliothèques canadiennes de l'époque, on sait moins ce qui advint d'elle après les événements précipités de 1760.

Ce n'est pas sans peine que l'on a pu reconstituer les linéaments de sa brève existence enfermée en quelque trente années. Assemblons quelques faits précis.

Elle était petite-fille de Simon Réaume ( $f l$. 1669-1723), marchand voyageur ${ }^{3}$ marié à Montréal en 1710 à Thérèse Catin

1 C. Tanguay, Dictionnaire... VI: 527.

2 Revue Canadienne, février, mars 1916.

3 A l'Inventaire de la communauté Réaume et Catin - une belle pièce de 70 pages - devant Gaudron de Chevremont, notaire, dressé du 29 mars au 10 avril 1734 (Archives judiciaires de Montréal, ci-après A.J.M.), il appert que Simon Réaume était commandant au poste des Ouyatanons, sans indication du lieu et de l'année de son décès. E. Voorhis dans son Historic Forts and Trading Posts... (Ottawa, 1930), 133, situe un poste de ce nom, rive nord de la Ouabache (Wabash), à 50 milles de Vincennes. Est-ce le même ? A cet inventaire, Ruet d'Auteuil apparaît débiteur de la succession pour 2.200 livres.

L'inventaire de la communauté Réaume et Catin révèle des biens meubles et effets de commerce, confondus dans la demeure et le magasin de la rue Saint-Paul, pour 11.080 livres, des créances pour 60.000 , et un passif de 24.000. Thérèse Catin-Réaume et ses enfants étaient donc restés en possession, à la mort du chef de famille, d'environ 47.000 livres, plus quelques immeubles. 
(1686-1763), dont le docteur Gabriel Nadeau 4 fait la bonne amie de Charles Ruet d'Auteuil (1690-1755) ${ }^{5}$ et qu'elle finit par épouser [1734]. Fille de Simon Réaume (1716-1760), elle était encore par sa mère, Louise de Couagne (1717-1756), petite-fille de René de Couagne (1690-1767), colonel des milices bourgeoises en 1760 , et l'un des premiers magistrats de Montréal, à titre de capitaine de milice, sous le régime militaire anglais (1760-1763).

Elle avait à peine dix-huit ans, en février 1760, quand on la maria, avec grandes pompes, à Joseph Fournerie de Vezon $(1729-1760),{ }^{6}$ de treize ans son aîné, ingénieur du roi, et fils d'un contrôleur général des fermes du roi à Metz.

Au contrat de mariage reçu le 2 février 1760, par Danré de Blanzy, contresignent, entre autres, le marquis de Vaudreuil, "grand'croix de l'ordre de Saint-Louis, lieutenant-général pour le Roy en toute la Nouvelle-France, terres et pays de la Louisiane », la marquise de Vaudreuil [née Charlotte Fleury d'Eschambault], François Bigot «chevalier, conseiller du roy,... intendant de justice, police et finances du pays », Mgr HenriMarie Dubreil de Pontbriand, «conseiller du roy en tous ses conseils, évêque de Québec », le chevalier de Lévis, « maréchal des camps et armées du roy, commandant en chef des troupes de sa Majesté dans l'Amérique septentrionale et chevalier de Saint Louis », M. de Bourlamaque, «brigadier des armées de Sa Majesté, chevalier de Saint-Louis », Messire Jacques-Joseph Guiton Monrepos, conseiller du roy, lieutenant général civil et criminel de la juridiction de Montréal, M. de Bougainville. Louise R. y était encore assistée de son père, Simon Réaume, de son aïeul maternel, René de Couagne, de sa grand'mère, Thérèse Catin, veuve d'Auteuil de Monceaux, de sa tante, Marguerite de Couagne, épouse de Michel-Louis de Parfourru, capitaine au régiment de Languedoc, de son oncle, Charles Réaume [1711-1781], ${ }^{7}$ de Simon

${ }^{4}$ Bulletin des Recherches historiques [ci-après BRH], (novembre $1935), 670$, note 2 .

5 C. Tanguay, Dictionnaire ..., 252. Correction: Charles d'Auteuil est décédé et inhumé à Chambly, et non à Saint-Antoine-sur-Richelieu, comme le dit Tanguay. N.B. Il y a d'autres cas où Tanguay a confondu SaintAntoine-sur-Richelieu avec Saint-Joseph de Chambly.

6 C. Tanguay, Dictionnaire ... IV: 498.

7 G. Nadeau, op. cit., 671; C. Tanguay, Dictionnaire... VI: 526. 
Sanguinet, ${ }^{8}$ de la belle Madame Péan, Angélique des Meloises, et de plusieurs autres.

Le régime y était la communauté de biens et le douaire de la mariée porté à 10.000 livres. Les biens de la future épouse sont décrits «tels qu'ils lui sont échus par la succession de sa mère ».

Simon Réaume, négociant, avait commandé les milices de Montréal au siège de Québec à l'été '59. Il y retourna avec le chevalier de Lévis au printemps de 1760 , mais il ne devait pas revenir de Sainte-Foy. En quatre mois, Louise R. perdit et son père et son mari.

Fournerie de Vezon, décédé à 31 ans, le 29 août 1760, pendant les dernières campagnes qui précédèrent l'encerclement de Montréal, fut inhumé dans la crypte de l'ancienne église de NotreDame de Montréal.

L'inventaire des biens de la communauté Fournerie et Réaume, dont Aegidius Fauteux n'indique pas la source, fut dressé par Pierre Panet, les 30 septembre et 2 octobre 1760 . Le praticien Panet donna par erreur à la veuve le patronyme [de Couagne] de sa mère, et confondit, en son acte, la relation des créances, considérables, de Simon Réaume avec celles de Fournerie.

Y figure en particulier l'intéressante bibliothèque décrite, en 1916, par Aegidius Fauteux, dans la Revue Canadienne. Contentons-nous d'y référer.

Cependant, peu de jours après la fermeture de l'inventaire (le 10 octobre, Panet) Louise Réaume disait renoncer à sa communauté de biens.

Veuve à dix-huit ans, après seulement six mois d'union, orpheline mais émancipée du fait de son mariage, de bonne race, riche de surcroît, Louise Réaume pouvait-elle demeurer longtemps seule? Elle ne devait pas tarder à refaire sa vie, selon le cliché cher aux veuves de guerre.

8 V. Mémoires, Société généalogique ... (janvier 1946), 26-29. 


\section{LE COLONEL DANIEL ROBERTSON (1733-1810)}

Où et quand contracta-t-elle un second mariage, vers 1761 , avec Daniel Robertson, pour lors chirurgien-major des troupes de l'occupation? Apparemment pas dans la région de Montréal. ${ }^{8 a}$

D'autre part, les registres tenus par les aumôniers ou pasteurs des occupants, et, plus tard, des anciens sujets de S. M. B., sont peu sûrs. Le registre de la première desserte protestante de Montréal, dont l'exemplaire déposé aux Archives du Palais de justice est une reconstitution datant d'après 1795 , n'est guère qu'un sommaire. Il ne s'ouvre que le 22 novembre 1766 , avec l'acte de mariage de deux francophones, Pierre-Paul Soubiran et Catherine-Félicité de Chaumont.

Quant au registre tenu par l'aumônier ambulant des troupes, le révérend Ogilvie, il n'a été retrouvé que récemment par George Home Wyrley Birch (1872-1954), patient chercheur, qui passa quarante ans aux Archives et dont les innombrables notes semblent être introuvables.

C'est au cours d'un voyage à New-York, vers 1920, que M. Birch retraça l'original de ce registre à la Trinity Church, la fameuse église de Wall Street. Il en obtint copie, qu'il déposa aux Archives de Montréal. Ce registre ne contient guère que des actes de baptême et de sépulture, pour un bon nombre, des rejetons illégitimes des officiers et troupiers de ce siècle où la laxité des mœurs était comme une règle d'élégance.

Ce que l'on sait, c'est que de l'union de Louise Réaume et de Daniel Robertson, chirurgien des troupes, naquirent et furent baptisés par le pasteur de la desserte protestante de Montréal:

a) le 4 mars 1767 , John;

b) le 4 avril 1768, Daniel, décédé le 6 septembre suivant;

c) le 13 mai 1770, James, décédé en bas âge ;

d) le 4 septembre 1771, Charlotte;

e) le 3 octobre 1773, Elisabeth;

f) Margaret, l'aînée, née en 1763, en lieux présentement inconnus.

8в Elle apparaît déjà remariée les 25 juin et 18 juillet 1761 (P. Panet, notaire) à la vente des effets et à la répartition des créances de son premier mari. 


\section{DECES DE LOUISE REAUME-ROBERTSON}

L'âge était dur. La tuberculose exerçait-elle ses ravages en cette famille ? La mère de Louise était décédée (1756) avant la quarantaine. Elle-même ne devait pas atteindre cet âge. Accouchée du 3 précédent, Louise Réaume s'éteignit le 17 octobre 1773, à l'âge de 31 ans. Elle alla rejoindre dans la crypte de l'église Notre-Dame, sa mère, son premier mari, et son aïeule, l'entreprenante Thérèse Catin.

Mais l'officiant à sa sépulture, dont la plume d'oie a bavé, a eu beau donner du «Monsieur » à son mari [Robertson], en dressant l'acte de décès, il faut presque deviner l'orthographe du nom du conjoint. Ce n'est que par recoupements, ainsi par l'âge de la défunte, que l'on peut tenir avec certitude qu'il s'agit bien, en cet acte, de l'épouse, en premières noces, de l'ingénieur [Fournerie de Vezon] qui possédait la plus belle bibliothèque de Montréal en 1760.

\section{DANIEL ROBERTSON}

\section{Commandant à Makinac, 1782.1787}

Un acte d'obligation pour 1.000 livres (sterling) à David Mitchell, chirurgien, signé à Michillimakinac, le 4 septembre 1782, et entériné par E.-W. Gray, notaire, à Montréal, le 2 septembre 1783, nous apprend que Daniel Robertson, L.S. (licensed surgeon) et pour lors commandant du poste de Michillimakinac devait être originaire de Dunkeld, comté de Perth, puisqu'il y donne hypothèque sur un patrimoine (estate) appelé Balmaguard.

Edwin O. Wood dans Historic Mackinac ${ }^{9}$ consigne que Daniel Robertson était entré dans l'armée en $\mathbf{1 7 5 4}$ et avait fait en Amérique les campagnes de la guerre de Sept ans. Licencié, il vécut, comme on l'a vu, à Montréal. Réengagé au $84^{\text {ème }}$ Régiment, à la faveur des événements de 1774 , c'est en 1782 qu'il succéda à Patrick Sinclair comme commandant à Makinac, poste qu'il remplit cinq années.

9 New-York, MacMillan, 1918. Note de Mlle Jacqueline Trépanier. 
En 1788 , il sollicitait ${ }^{10}$ pour ses quatre enfants des concessions de terre. Il reçut quelque 3.000 acres dans le canton de Chatham, sur la rive de l'Outaouais.

Promu lieutenant-colonel en 1794 , il devint, en 1798, colonel du deuxième bataillon du $60^{\mathrm{e}}$ Régiment, le Royal American.

Le 31 mars 1806, devant J.-A. Gray, notaire, de Montréal, il faisait une donation de terre, dans le canton de Chatham, au révérend Richard Bradford.

Le 30 mai 1809, devant le même Gray, il dictait un testament et il y nommait Niel Robertson (1760-1813), de Côteaudu-Lac, - son frère ? - son exécuteur testamentaire. Il le révoquait à ce titre en mai suivant.

Le 18 décembre 1809, le colonel Daniel Robertson, de Chatham, malade, en séjour chez son gendre, Daniel Sutherland, rue Saint-Paul à Montréal, dictait un dernier testament. Il n'y nommait pas son fils, non plus que ses filles, Margaret et Charlotte. Il n'en désignait qu'une, Elisabeth, mais sans lui faire de legs. Il instituait ses deux petites-filles, Maria Sutherland, épouse de James Hallowell, marchand, et Louisa Sutherland (née en 1799), ses légataires universelles, la première pour un quart de sa succession, et la seconde pour les trois-quarts. Les exécuteurs testamentaires sont Daniel Sutherland, précité, et Robert Griffin, seigneur de Nazareth ${ }^{11}$ et dont le nom est passé à un quartier de Montréal, Griffintown.

Le colonel D. Robertson s'éteignit à Montréal le 5 avril 1810 . Le pasteur Somerville, de l'Église d'Écosse, dressa son acte de sépulture.

L'inventaire de sa succession, dressé le 22 juin 1810, par J.-A. Gray, notaire, révèle des biens meubles, en la maison de D. Sutherland, pour 71 livres (sterling) et shillings; en espèces, $£ 79$; des créances pour $£ 150$; des cens et rentes dus par 22 concessionnaires, y nommés, du canton de Chatham pour $£ 206$; l'acte de concession (patent) au canton de Chatham, en date du

${ }^{10}$ Etats de service militaire, concessions de terre et nominations diverses de Daniel Robertson. Notes de M. Pierre Brunet, sous-ministre adjoint, Archives du Canada.

11 E.-Z. Massicotte, BRH, (janvier 1945), 73. 
31 décembre 1806, à D. Robertson conjointement avec Simon Fraser, dont 3.000 acres au nom de D. Robertson et 2.000 à S. Fraser; un acte de concession de 200 acres au canton Crosby, Haut-Canada, au nom de M. Green.

La vente des effets, dont procès-verbal suit l'inventaire, rapporta $£ 75$.

Le 10 août 1810, encore devant J.-A. Gray, notaire, de Montréal, l'exécuteur testamentaire de D. Robertson, son gendre, Daniel Sutherland, au surplus autorisé en justice, procédait à la vente de l'immense concession de Robertson dans le canton de Chatham, au révérend Richard Bradford, pasteur de l'église du Christ à Sorel. A noter en cet acte qu'il est mention que Daniel Sutherland avait acquis les 2.000 acres concédés en ce canton conjointement à Simon Fraser et à Robertson. La vente comprend les 5.000 acres de la concession, dont l'acte est de décembre 1806. Elle est faite pour le montant de 2.500 livres (sterling).

\section{LA DESCENDANCE DE LOUISE REAUME-ROBERTSON}

Le fils aîné de Louise Réaume et de Daniel Robertson, John Robertson (né en 1767), pour lors lieutenant au $60^{\mathrm{e}}$ Régiment, contracta à l'église du Christ, de Montréal, le 10 janvier 1793, un beau mariage avec Catherine Christie, née en 1772, fille du général Gabriel Christie, devenu, par ses achats successifs de fiefs, le plus grand propriétaire de seigneuries de la province.

Sa fille aînée, Margaret (née en 1763), épousa devant le pasteur de la desserte protestante de Montréal, le $1^{\text {er }}$ septembre 1781, Daniel Sutherland. Elle mourut à Montréal le 3 avril 1838.

Daniel Sutherland, l'un des organisateurs d'un service d'eau à Montréal en 1800, maître de poste à Montréal en 1807, fut major en 1813 au premier bataillon de Montréal, dont James McGill était le colonel commandant. Lieutenant-colonel d'état major en 1815, il devint directeur général adjoint de la poste en 1816. Syndic de l'Institution royale pour l'enseignement en 1824 , il démissionna de ses fonctions à la poste en $1827 . .^{12} \mathrm{Il}$ mourut avant 1836.

${ }^{12}$ F.-J. Audet et E. F.-Surveyer, Les Députés au 1er Parlement [1792] (2 vol., Montréal, 1946), I: 221. 
Ajoutons que Maria Sutherland-Hallowell fut - détail inédit - la mère de Louise Hallowell-Pinsoneault (1816-1860), née à Brookline, New-Hampshire, mariée en 1839 à Alfred Pinsoneault (1812-1874), avocat de Montréal, propriétaire d'un grand domaine à la Tortue (Laprairie) et seigneur de Léry (Napierville)..$^{13}$

La deuxième, Charlotte Robertson, née en 1771, épousa à 20 ans, en l'église du Christ à Montréal, le 15 mars 1792, le docteur John Farries. Décédée à Montréal le 8 janvier 1826, son mari lui survécut.

\section{LES HERTEL}

La dernière, Élisabeth (née en 1773) épousa en l'église Notre-Dame de Montréal, le 19 mars 1794, Hippolyte Hertel ( $f l$. 1771-1802), né à Montréal, plus tard lieutenant aux Volontaires Royaux, fils d'Hippolyte Hertel (1738-1781), capitaine au Département des Sauvages et de Marie-Anne Lecomte-Dupré (1740-1792). C'est cette dernière qui avait cédé, en 1792, le terrain où s'éleva la première église des Écossais à Montréal, qui prit le nom de la rue où elle donnait, Saint-Gabriel, et qui fit place plus tard à l'annexe de l'ancien Palais de justice. ${ }^{14}$

Petit-fils de Joseph Hertel ( $f l$. 1701-1752), seigneur de Pierreville, le marié de 1794, Hippolyte Hertel, était le cousin germain, entre autres, de Charles-Michel de Salaberry, le héros de Châteauguay. Le contrat de mariage, reçu par Chaboillez, fut passé le même jour en l'hôtel de John Campbell (1730-1795), colonel du Département des Sauvages. ${ }^{15}$ Le douaire de la mariée y était de 30.000 chelins (shillings).

Du mariage d'Élisabeth Robertson et d'Hippolyte de Hertel naquirent, selon P.-G. Roy, trois enfants :

Jean-Hippolyte (1795-1822), né et décédé à Montréal, officier aux Voltigeurs pendant la guerre de 1812-1813;

Daniel, né à Québec en 1797 ; 1952), 173.

14 Robert Campbell, A History of the Scotch Presbyterian Church, St. Gabriel Street, Montreal (Montreal, Drysdale, 1887), 65.

15 Rapport de l'Archiviste de la Province (1947-1948), 32. 
enfin, Louis-Joseph, sans indication de lieu ni de date de naissance.

Ici, une rectification. P.-G. Roy dans son étude sur les Lecomte-Dupré a confondu deux Daniel de Hertel, en faisant marier, en 1843, à Montréal, Daniel, né à Québec en 1797. Au surplus, il ne semble pas que Hippolyte Hertel et Élisabeth Robertson eurent un troisième fils, prénommé Louis-Joseph.

Leur union ne fut pas heureuse. Le 17 février 1806, dans une requête, rédigée en français, en tutelle aux mineurs ${ }^{16}$ issus de leur mariage, il est question de leurs deux fils, Jean et Daniel. Le requérant est l'aïeul maternel, Daniel Robertson, colonel du $60^{\text {ème }}$ Régiment. ${ }^{17}$ Il allègue que le père est absent de la province depuis trois ans, et que depuis, sa fille, Élisabeth, a contracté un autre mariage aux États-Unis. Il s'agit sans doute de l'union d'Élisabeth Robertson avec Théodore Davis (1778-1841), arpenteur, de Saint-André d'Argenteuil, originaire du New-Hampshire, que mentionne Cyrus Thomas, l'annaliste d'Argenteuil. ${ }^{18}$

De fait, le dossier de la tutelle comporte un procès-verbal, signé $\mathrm{P}$. Lukin, de signification de la requête, à Élisabeth Robertson «en sa demeure, au village de Saint-André d'Argenteuil ».

Au conseil de famille, apparaissent, entre autres, Hippolyte Saint-Georges-Dupré, ${ }^{19}$ cousin-germain paternel, et Daniel Sutherland, précité, «oncle maternel ». Dupré est élu tuteur. Le 4 juin suivant, Michel Dumas était nommé curateur aux biens d'Hippolyte Hertel.

La maison de Théodore Davis et d'Elisabeth Robertson, à Saint-André, plus tard passée au fils de celle-ci, Daniel de Hertel, était occupée, en 1895, par Ronald-Gaspard [Denys] de La Ronde (1841-1924), avocat. $^{20}$

16 P.-G. Roy, La Famille Lecomte-Dupré (Lévis, 1941), 48.

17 Cour du Banc du roi, A. J. M.

18 C. Thomas, History of the Counties of Argenteuil. Qué. and Prescott, Ont., (Montréal, 1896), 180.

19 BRH (décembre 1953), 199.

20 Cyrus Thomas, op. cit., 80, 95, 127; J.-Edmond Roy, Histoire $d u$ Notariat au Canada, II : 309-313. 


\section{LE COLONEL DANIEL DE HERTEL (1797-1866)}

Daniel de Hertel, né à Québec en 1797, engagé, encore adolescent, dans les armées, lieutenant aux Voltigeurs dans la même compagnie que son frère aîné, Jean, pendant la guerre de 1812$1815,{ }^{21}$ participa aux actions de Crysler's Farm et de Plattsburg.

Le 17 août 1818, il épousait à l'église anglicane Saint-André d'Argenteuil ${ }^{22}$ Lydia Brown, fille de John Brown, papetier et éditeur, de Montréal. C'est le premier mariage jamais célébré en cette congrégation.

Le 7 septembre suivant, il a la qualité de capitaine de milice, alors qu'il signe, à Rigaud, comme témoin à la sépulture d'une immigrante irlandaise de 26 ans, dont l'officiant, l'abbé La Broquerie, épelle le patronyme Benen [Marie]. A Rigaud encore, le 25 janvier 1824, il est parrain de Daniel Guertin, fils de Pierre Guertin et d'Abigaïl Warren, d'Argenteuil.

Le 14 octobre 1828, sa fille, Frances, est baptisée à SaintAndré d'Argenteuil, dans le rite anglican. Elle décède le 26 décembre suivant.

En septembre 1827, il devenait lieutenant-colonel du $2^{\mathrm{e}}$ bataillon de milice d'York (Argenteuil). Il avait trente ans. Daniel de Hertel a cette qualité au baptême, célébré selon le rite romain, à Rigaud, le 28 août 1831, de son fils, Jean-Édouard, né le 6 juillet précédent, et dont le parrain est Alexis-Édouard Montmarquet, marchand, l'un des pionniers de Saint-André et l'un des requérants à la constitution de la paroisse en $1835 .^{23}$

Daniel de Hertel fils, qui se maria à Montréal en l'église du Christ, le 6 mars 1843, à Sophia Cameron, était-il le fils du colonel ? Fort probable, d'autant que le contractant, dont l'officiant ne donne pas la filiation, est déclaré de Chatham, soit, sauf rectification, le canton où a été érigée plus tard la paroisse SaintPhilippe d'Argenteuil.

Quant aux autres enfants que lui attribue Pierre-Georges Roy, ${ }^{24}$ Louis-Théodore, Frances, mariée à John Fraser, et Marie-

${ }^{21}$ L. Homfray Irving, British Officers of the War of 1812-1815, (Toronto, 1908).

22 C. Thomas, op. cit., 108.

23 C. Thomas, op. cit., 115.

24 P.-G. Roy, passim, 49. 
Louise, mariée à H. W. Robertson, sans indication de lieu ni de date de naissance ou de mariage, il n'y a pas d'autres sources connues présentement.

Robert de Roquebrune, en son étude sur les Hertel, parue dans Nova Francia, ${ }^{25}$ n'est pas plus précis. Son délicieux Testament de mon enfance, ${ }^{26}$ fait encore mention de $"$ Hertel, anglais, inconnus », qui surgissent soudain à un enterrement.

Dans ses souvenirs, le major A. McMillan dit Daniel de Hertel présent à Sainte-Scholastique, en décembre 1837. ${ }^{27}$ Il n'y a pas de présomption à tenir Hertel pour loyaliste...

Enfin, sous l'empire de la loi nouvelle de l'enregistrement des immeubles, décrétée par le régime de l'Union (1841), Daniel de Hertel devint le premier registrateur d'Argenteuil, à SaintAndré, poste qu'il remplit jusqu'au transfert du chef-lieu à Lachute.

Daniel de Hertel est décédé soudainement, au printemps de 1866 , après avoir passé en revue plusieurs compagnies de milice d'Argenteuil, en partance pour la frontière que menaçaient de franchir les Fenians de l'État de New-York. ${ }^{28}$

L'annaliste C. Thomas le décrit comme «un bel homme, de plus de six pieds de taille, à l'air martial». Peut-être tenait-il pour quelque peu cette prestance imposante de son bisaïeul, Simon Réaume, tombé au champ d'honneur, à Sainte-Foy, un siècle auparavant?

Le monument érigé aux morts de la grande guerre de 1914, complété depuis avec ceux de la guerre de 1939, par la Ville de Westmount, place de l'hôtel de ville, face à la splendide église paroissiale de l'Ascension, contient au panneau-est, entre autres, les noms de Charles-P. Massy et Hugh de Hertel Massy.

\section{LES MASSY}

Deux frères, portant les mêmes prénoms, Joseph Massy (1701-1766) et Joseph Massy ( $f l .1702-1769)$, natifs de Québec, ${ }^{29}$

25 1926, 60-66.

26 Montréal, la Palatine, 1951.

27 Cyrus Thomas, op. cit., 369.

28 C. Thomas, op. cit., 126-127.

29 C. Tanguay, Dictionnaire... V : 565. 
épousèrent à Montréal, respectivement en 1726 et 1729, les deux sœurs, originaires de Montréal, Hélène Bardet-Lapierre (17061777) et Marie-Josephte Bardet-Lapierre (1713-1755) .

Du mariage du premier Joseph $M$. à Hélène Bardet naquit, entre autres, Joseph Massy (1728-1758) qui, ordonné prêtre récollet, en 1755, devint aumônier des troupes et mourut au fort Carillon [Ticonderoga] le 10 novembre $1758 .{ }^{30}$

Il y a trace que des descendants des deux frères Massy, établis à Montréal en 1760 , se retrouvaient un siècle plus tard, dans Deux-Montagnes et Argenteuil.

Se peut-il que par le jeu des alliances, Hugh de Hertel Massy, tombé sur les champs de bataille en 1916 ou en 1917, fut, à la fois, le descendant de l'un de ces frères Massy et de l'un ou des Daniel de Hertel, mariés respectivement en 1818 et en 1843 ? ${ }^{31}$

Ainsi se clôt, sous le signe de Mars, la descendance connue de Louise Réaume, la mariée de février de 1760, dont la noce avait réuni, peu avant qu'elle ne s'efface à jamais, l'élite sociale de la Nouvelle-France.

JEAN-JACQUES LEFEBVRE

30 Notes du Révérend P. Archange Godbout, o.f.m.

31 Cet article était composé quand le hasard des recherches mit sur la trace de l'identité de Hugh de Hertel Massy ( $f l .1895-1915)$. Il était fils de George Hugh Massy ( $f l$. 1850-1912) natif d'Irlande, ingénieur, venu au Canada en 1871 et décédé à Montréal. G.-H. Massy dirigea (1885) l'érection du pont du chemin de fer du Pacifique entre Lachine et le Sault-Saint-Louis (vulgo Caughnawaga). Il avait épousé à Montréal, en 1880, Georgina Fenwick (1852-1920), fille du Dr George E. Fenwick ( $f l .1824-1894)$, professeur de chirurgie à la Faculté de McGill et de Charlotte Hertel (18231907), et petite-fille du colonel Daniel de Hertel. Engagé (1914) au $7 e$ Régiment de la Colombie britannique, passé outremer avec le premier contingent canadien, Hugh de $\mathrm{H}$. Massy fut tué au feu d'Ypres en avril 1915. Colonel William Wood, The Storied Province of Quebec, (1931), IV: 345. 